\title{
Research on High Reliability Power Supply Design Scheme of Nuclear Power Plant
}

\author{
WANG Geng ${ }^{1, *}$ \\ ${ }^{1}$ CNPDC, State Key Laboratory of Nuclear Power Safety Monitoring Technology and Equipment, 518172, Shenzhen, Guangdong, China
}

\begin{abstract}
After the Fukushima nuclear accident, the reliability requirements for Nuclear Power Plant (NPP) safety systems have been further improved worldwide. Therefore, it is necessary to provide a safe, reliable and economical scheme of the power supply system to cope with the abnormal conditions. Based on the reliability of the power supply of the 3rd generations of NPPs and combined with the application of the defend in depth concept in the electrical system, this paper provides a brief introduction of the typical 3rd generation NPP electrical system in the following area: the configuration of the electrical power system, defence in depth principle of the power supply, the basic structure of electrical power system. On this basis, an optimal power supply scheme is proposed.
\end{abstract}

\section{Background}

Nuclear power plant (NPP) is an important way to achieve low-carbon power generation and it is a green energy source. However, after the Fukushima nuclear accident, the safety of NPPs was once again widely questioned by public opinion and the reliability requirements for NPP safety systems have been further improved worldwide $[1,2]$.

The power supply system and distribution system of NPPs consist of the auxiliary electrical system that provides reliable and safe power supply to all of the equipment in the plant. The power supply sources are necessary for safety systems and equipment of NPPs and play an important role in performing their safety functions during normal operating conditions, accident conditions and after accident conditions. Therefore, it is necessary to provide a safe, reliable and economical scheme of the power supply system to cope with the abnormal conditions [3, 4].

Currently, the 3rd generation NPPs usually rely on switching standby power supply, emergency diesel generators (EDG), SBO diesel generators, short-term battery(such as 2 hours), long-term battery(such as 12, 24 or more hours) and mobile diesel generators to provide power to NPPs in abnormal conditions, thereby improving the safety and reliability of NPPs. However, diesel generators and batteries have some shortcomings such as high idleness ratio, high cost of maintenance, high cost of regular test, high cost of qualification and high cost of the manufacture. The capability to response to floods, tsunamis and other external hazards is weak.
The human sources for maintenance and operation are hard to control. Hence, an optimal power supply scheme is necessary to be proposed.

\section{Brief introduction of electrical power supply in 3rd generation NPP}

\subsection{Configuration of the electrical power system} In 3rd generation NPPs, according to the different technical routes, there are different numbers of the safety trains and different measures to cope with the Design Extension Condition (DEC) condition. But the design principles of the electrical power system are basically similar. Generally, the design of electrical power system is based on the requirements of the process systems and the defence in depth principle.

The typical configuration of 3rd generation NPPs is shown as the Fig. 1. The electrical power system of the NPPs can be divided into off-site power system and onsite power system. The off-site power system includes the main power from the external grid 1 (such as $500 \mathrm{kV}$ ) and standby power from the external grid 2 (such as $22 \mathrm{kV}$ ). The on-site power system includes main generator, emergency diesel generators (EDG), SBO Diesel Generators (SBO DGs), short-term battery (such as 2 hours), long-term battery (such as 12, 24 or higher hours) and mobile DG. And the off-site power system and the main generator together constitute the preferred power supply. This typical configuration of the electrical power system is based on the defence in depth principle.

Corresponding author: 182505810@qq.com 


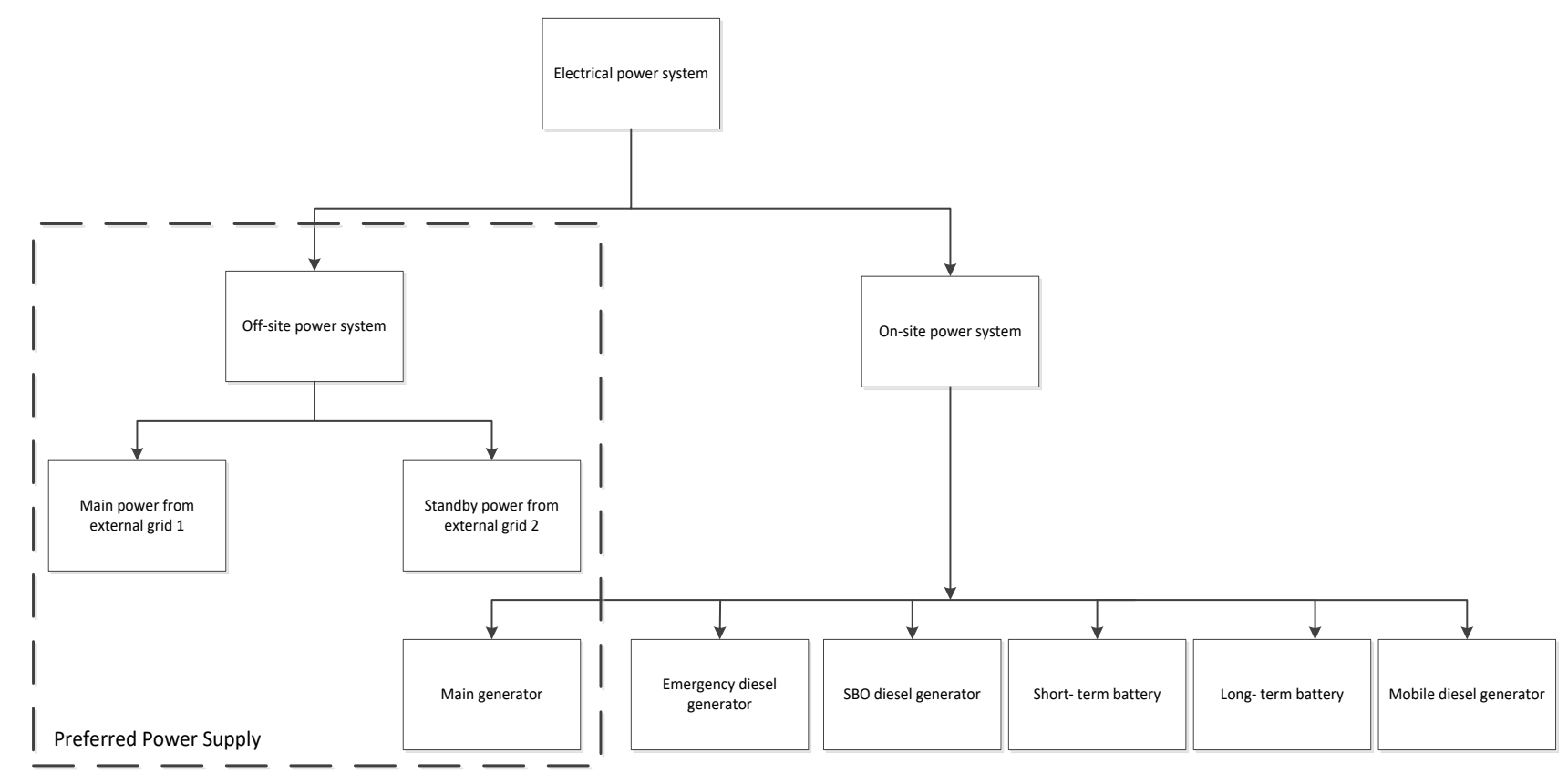

Fig. 1. Configuration of the Electrical Power System

\subsection{Defence in depth of electrical power system design}

The defence in depth of electrical power system design is based on the codes and standards of national and international, such as HAF102 [5] and IAEA SSG 34 [6]. According to the requirements of the codes and standards, the intended electrical power system approach to defence in depth is as follows:

a) Normal operation: The off-site grid or the main generator provides a power supply to the normal power distribution system. They also supply the emergency and SBO power distribution systems, keep the DC batteries charged and provide power to the loads which are powered from the emergency or SBO power distribution system during normal operation. Electrical protection systems, a standby grid connection and the ability to transfer to house load are provided to minimise the progression from abnormal operations to faults.

b)Design basis faults: The systems delivering the main line of protection are powered from the emergency power distribution systems, with power supply from the grid (if available), from the main generator (if available) or from the EDGs. For frequent faults where a second line of protection is required, the systems delivering the second line are powered from the SBO power distribution systems, with power supply from the grid (if available), from the main generator (if available), from the EDG (if available) or from the SBO Diesel Generators. For DC and AC Uninterruptible Power Supply (UPS) systems, the current design is that the short-term battery system (NI $2 \mathrm{~h}$ DC and AC UPS) provides power supply to both main line and second line equipment.

c) Beyond design basis faults and severe accidents: Where systems are provided to mitigate beyond design basis faults, these may be powered from either the emergency or SBO power distribution systems, depending on the detail of the fault. Connections are provided for mobile generator power supply to mitigate loss of grid and all on-site generation sources. Systems provided to mitigate severe accidents are powered by the long-term battery system (NI 12h DC and AC UPS).

\subsection{Structure of the electrical power system}

\subsubsection{Preferred Power Supply}

The normal conditions include the conditions during plant power operation, start up and normal shutdown. During plant power operation, the power generated by the main generator is transmitted to the external main grid through the unit transformer. Meanwhile, the unit auxiliaries are powered by the main generator as well. The main connection is also in service during plant startup and normal shutdown.

If there is a fault with the off-site main power supply and meanwhile the generator is available during normal conditions, the plant will transfer to the house load condition, in which the generator provides power supply to the plant auxiliary equipment.

The generator set can be tripped in the event of the turbine unit tripping, or the reactor tripping, or an electrical fault of the main generator. In such cases, the plant will be fed by the off-site main power supply.

During plant normal and transient conditions, the standby connection system is in backup condition. When the main connection is unavailable due to a fault condition and the house load operation is not successful, the automatic switchover between the main connection and standby connection is executed to ensure the continuous power supply for the unit to shut down.

\subsubsection{The EDG}


During plant normal and transient conditions, the EDG is in the stand-by state and its pre-treatment system is in operation. When the off-site power system and the main generator are failure, the EDGs provide power to the onsite emergency distribution system in the event of Loss of Offsite Power (LOOP).

\subsubsection{The $S B O D G$}

During plant normal, transient conditions and DBC condition, the SBO DG is in the stand-by state and its pre-treatment system is powered. In case of a SBO condition and LOOP combined with all EDGs failures, the SBO DGs could start up and connect to the NI medium voltage SBO power distribution system to cope with the SBO condition.

\subsubsection{The Short-term Battery}

During normal operation condition, the charger of the short-term battery system (NI $2 \mathrm{~h}$ DC and AC UPS) rectifies the $\mathrm{AC}$ power to $\mathrm{DC}$ power, provides power to loads and charges the battery. The battery is kept at full charge by the float charge. The inverter converts the DC power to three-phase or one-phase AC power. The bypass circuit is in a standby state. The regulated power supply system provides a power supply to the load with the regulating transformer which is supplied by the $\mathrm{AC}$ emergency power system.

During transient conditions, the short-term battery system (NI 2h DC and AC UPS) is in service and is powered by the emergency AC power distribution system. In case of losing $\mathrm{AC}$ power, the battery will provide a power supply to the loads. After AC power recovery, the battery charger will provide power supply to the loads and charge power for the battery. In case of short-circuit, the failure will be removed selectively and the system will restore operation after proper maintenance.

In the LOOP condition, the EDGs start-up and provide power to the short-term battery system (NI $2 \mathrm{~h}$ DC and AC UPS). Before the EDGs start-up, the shortterm battery system (NI $2 \mathrm{~h}$ DC and AC UPS) provides the power.

In the LOOP condition cumulated with failure of the EDGs, the SBO DGs start-up and provide power to the short-term battery system (NI $2 \mathrm{~h}$ DC and AC UPS) except for the regulated power supply system. Before the SBO DGs start-up, the short-term battery system (NI $2 \mathrm{~h}$ $\mathrm{DC}$ and AC UPS) provides the power.

\subsubsection{The Long-term Battery}

During normal operation condition, the charger of the long-term battery system (NI $12 \mathrm{~h}$ DC and AC UPS) rectifies the $\mathrm{AC}$ power to $\mathrm{DC}$ power, provides power to loads and charges the battery. The battery is kept at full charge by the float charge. The inverter converts the DC power to three-phase or one-phase AC power. The bypass circuit is in a standby state.

During transient conditions, the long-term battery system (NI 12h DC and AC UPS) is in service and powered by the SBO AC power distribution system. In case of losing $\mathrm{AC}$ power, the battery will provide a power supply to the loads. After AC power recovery, the battery charger will provide a power supply to the loads and charge power for the battery. In case of short-circuit, the failure will be removed selectively and the system will restore operation after proper maintenance.

In the LOOP condition, the EDGs start-up and provide power to the long-term battery system (NI $12 \mathrm{~h}$ DC and AC UPS). Before the EDGs start-up, the longterm battery system (NI 12h DC and AC UPS) provides the power.

In the LOOP condition cumulated with failure of the EDGs, the SBO DGs start-up and provide power to the long-term battery system (NI 12h DC and AC UPS) except for the regulated power supply system. Before SBO DGs start-up, the long-term battery system (NI 12h $\mathrm{DC}$ and AC UPS) provides the power.

The SBO condition cumulated with failure of the SBO DGs are considered in the power balance for batteries of the long-term battery system (NI 12h DC and AC UPS).

\subsubsection{The Mobile DG}

During plant normal, transient conditions and DBC condition, the mobile DG is out of service.

In the DEC conditions where the stationary DGs (SBO DG or EDG) are available, the mobile DG is not needed. In the condition of a total loss of AC power supply, the mobile DG is driven to the outside of the NI building and connected to the fast-connection box. The operator manually starts the mobile DG when the connection is complete.

The mobile DG breaker can be closed if the frequency and voltage of the generator reaches the nominal value. The loads can then be loaded to the mobile DG manually.

\section{Optimal power supply design scheme in NPP}

As described in the introduction of electrical power supply in 3rd generation NPP, the EDGs, SBO DGs, short-term battery system, long-term battery system and mobile DG play an important role in the abnormal conditions, which especially beneficial for improving the safety and reliability of the power source. However, diesel generators and batteries have some shortcomings. And the capability to response to floods, tsunamis and other external hazards is weak. Therefore, this paper provides an optimal emergency power supply scheme which uses the wind, solar and water energy as the backup power supply. This optimal emergency power supply system can provide AC and DC power which could be backup power source for part of the diesel generators or battery systems, or instead of part of the emergency power system. The initial scheme of the optimal emergency power supply system is shown as the Fig. 2.

The configuration of the optimal emergency power supply system mainly includes: 
a) The energy storage tower building which is construction of the optimal emergency power supply system;

b)Unit controller which is used for inspecting and controlling the operating condition of the emergency power supply;

c) Small wind and solar energy storage power supply system (includes small wind energy storage system, small solar energy storage system and batteries) which provides power supply to the unit controller, short-term battery system and long-term battery system;

d)Water energy storage system which transforms the water storage energy to the electric power according to the command of the unit controller.

The operation of the optimal emergency power supply system is shown as the Fig. 3 .

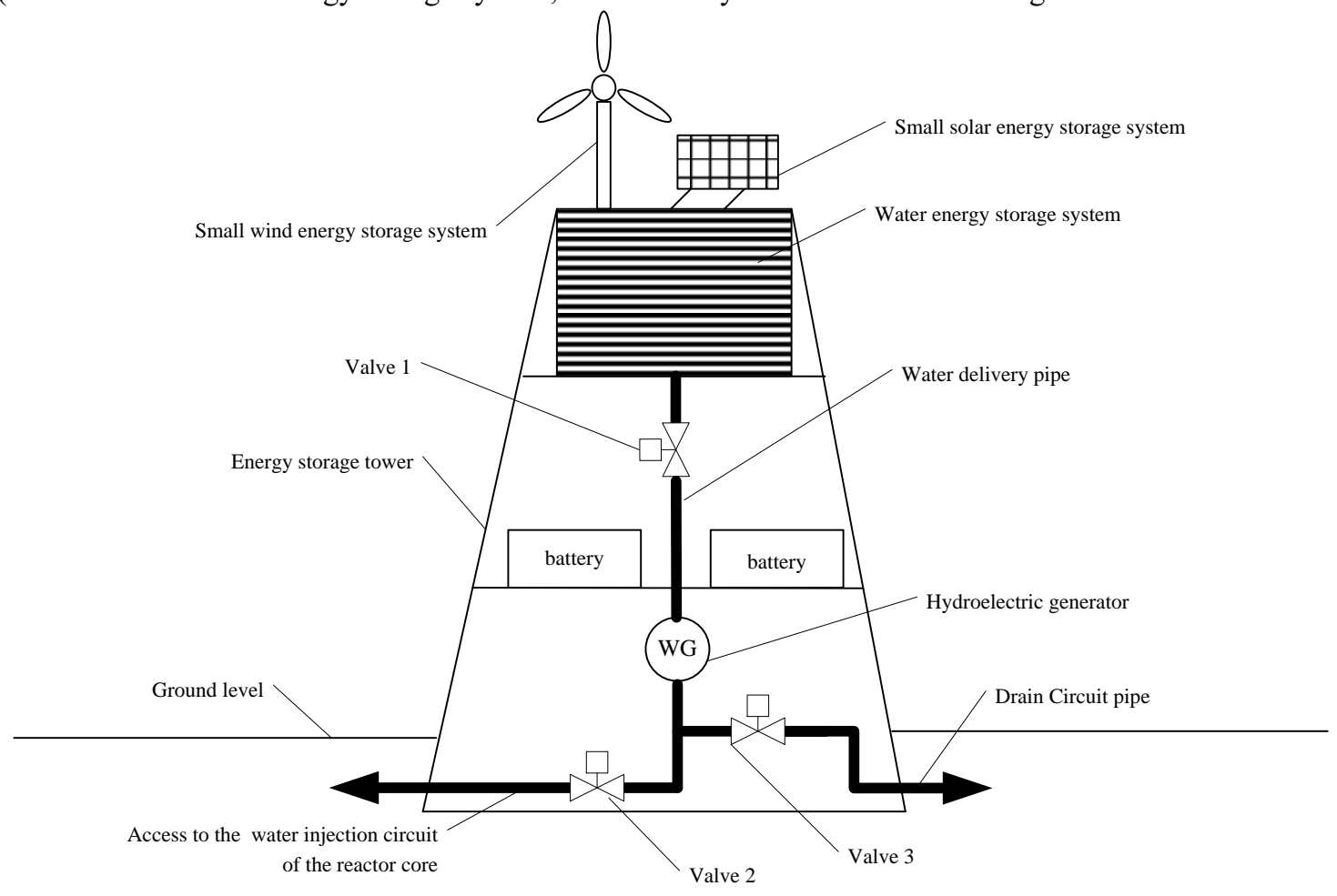

Fig. 2. The Initial Scheme of the Optimal Emergency Power Supply System

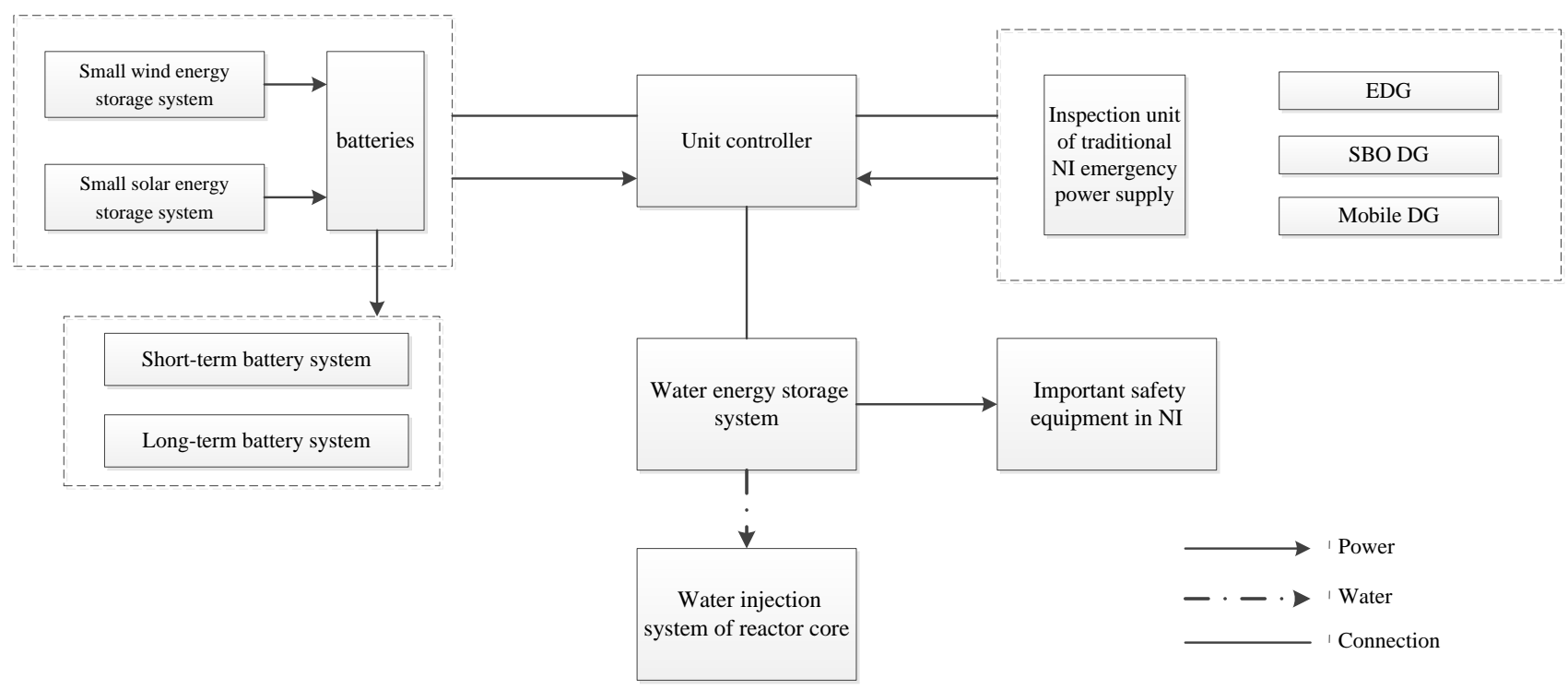

Fig. 3. The Operation of the Optimal Emergency Power Supply System

During plant normal and transient conditions, the optimal emergency power supply system is standby. Small wind and solar energy storage power supply system is continuously operating and storages the energy to the batteries of optimal emergency power supply system. The batteries are kept at full charge by the float charge and provide the control power to the unit controller. There has an inspection signal from the traditional emergency power supply of NPP to the unit controller of the optimal emergency power supply system. According to the design requirements of the specific NPP project, the optimal emergency power supply system can 
be supplementary or backup power source for EDGs, SBO DGs and mobile DG. When the traditional emergency power supply systems are unavailable, the unit controller will order the water energy storage system to provide power supply and transfer the power supply to the important safety equipment in NI. For example, if the optimal emergency power supply system is a backup power supply for SBO DGs, the operational process is mainly as follows:

During the SBO condition, when the EDGs are failure and the SBO DGs could start up, the optimal emergency power supply system is standby. When the EDGs and SBO DGs are failure, the optimal emergency power supply system could start up and connect to the NI medium voltage SBO power distribution system to cope with the SBO condition. If the SBO DGs or EDGs is recovery, the optimal emergency power supply system is back to standby. The SBO DGs or EDGs provide power supply to the safety systems and equipment continuously. In specific severe accident condition, if it is necessary, the controller could control the water from the water energy storage system delivery to the water injection circuit of the reactor core as emergency cooling water.

\section{Conclusion}

This paper summarized the configuration of the electrical power system, defence in depth of electrical power system design and typical structure of the electrical power system in the 3rd generation NPP. In order to improve the safety, reliability and diversity of the electrical power supply of NPP, an optimal power supply design scheme has been provided.

For the optimal power supply design scheme, initial scheme of the optimal power supply system has been provided. A brief description, configuration and operation example of the optimal power supply design scheme have been shown as a reference for the design of engineer project.

\section{References}

1. ZHAO Ran. Reliability Analysis of Electric Power System for Large -scale NPP. Industrial \& Science Tribune, 2018, (19).

2. MEI Qizhi. Reliability Analysis of Electric Power System for Large -scale NPP. NUCLEAR POWER ENGINEERING , 1994 (06), 486 492.

3. Ran Huimin. Reliability Application of Electric Power System in NPP. Electrotechnical Application. 2006 (08), 22 25.

4. YANG Zhihui, ZHANG Tao. Study on Improvement for Service Power Supply for a Nuclear Power Plant. Nuclear Science and Engineering, 2012, (32), 24 30.

5. National Nuclear Safety Administration. NUCLEAR POWER PLANT DESIGN SAFETY REGULATIONS HAF 102, 2016.

6. IAEA International Atomic Energy Agency. Design of Electrical Power Systems for Nuclear Power Plants. NO. SSG 34, 2016. 